\title{
Modeling performance of sample collection sites using whole exome sequencing metrics
}

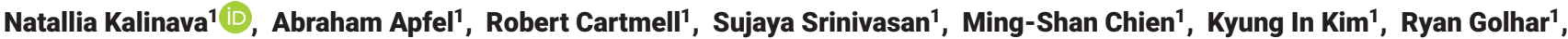
Kathryn E Bednarz ${ }^{1}$, Saumya Pant ${ }^{1}$, Joseph Szustakowski ${ }^{1}$, Scott D Chasalow ${ }^{1}$, Ariella Sasson ${ }^{1}$ \& Stefan Kirov ${ }^{*, 1}$

${ }^{1}$ Bristol Myers Squibb, Princeton, NJ 08648, USA; *Author for correspondence: stefan.kirov@bms.com

BioTechniques 69: 421-426 (December 2020) 10.2144/btn-2020-0086

First draft submitted: 10 June 2020; Accepted for publication: 28 August 2020; Published online: 26 October 2020

\section{ABSTRACT}

Although next-generation sequencing assays are routinely carried out using samples from cancer trials, the sequencing data are not always of the required quality. There is a need to evaluate the performance of tissue collection sites and provide feedback about the quality of next-generation sequencing data. This study used a modeling approach based on whole exome sequencing quality control (QC) metrics to evaluate the relative performance of sites participating in the Bristol Myers Squibb Immuno-Oncology clinical trials sample collection. We identified several events for the sample swap. Overall, most sites performed well and few showed poor performance. These findings can increase awareness of sample failure and improve the quality of samples.

\section{METHOD SUMMARY}

We utilized the whole exome sequencing quality control (QC) metrics from samples of Bristol Myers Squibb Immuno-Oncology clinical trials as a performance indicator. The QC step of the sample - genetic matching between the tumor and the blood samples from the same patient defined two sources of potential sample failure: sample swap and cross-contamination. We performed Bayesian logistic regression to assess the relationship between QC success rate and relative site performance, adjusting for indication.

\section{KEYWORDS:}

collection site $\bullet$ modeling performance $\bullet$ NGS $\bullet$ sample QC $\bullet$ whole exome sequencing

Emerging technologies in healthcare, such as next-generation sequencing (NGS), require continuous improvement. Clinical laboratories collect, process and store tissue samples based on recommended best practices and standard protocols. The National Cancer Institute's Best Practices for Biospecimen Processing and WHO's Good Clinical Laboratory Practice (GCLP) are intended to promote the quality and validity of data generated from samples collected in clinical trials [1,2]. These guidelines describe the process and conditions under which laboratory studies are planned, performed, monitored, recorded and reported (OECD GCLP Guideline). The performance of clinical laboratories in the USA is overseen by regulatory agencies including the Institute of Medicine, Agency for Healthcare Research and Quality, the Centers for Medicare and Medicaid Services and the National Committee for Quality Assurance. However, clinical trials often include sites from multiple countries. In that case, studies must be compliant with International Conference on Harmonization guidelines and the Declaration of Helsinki, as well as local laws and regulations [3-5].

As genomic sequencing technologies become more affordable and the assays stabilize and improve, genetic tests in clinical practice increase in a growing number of countries for a wide range of conditions. It is essential that this increased use is accompanied by the appropriate oversight. Feedback about the quality of genetic data generated from samples at various sites will help laboratories optimize their processes and improve the reliability and clinical utility of the data. Efficient quality assessment depends on good quality indicators, a practical measurement that can be easily obtained and reproduced [6]. While there are recommendations for genetic testing [7,8] and regulatory guidelines for the transfer and protection of genetic results $[9,10]$, it is still an open question how to validate and globally track the performance of clinical laboratories for genomic assays, particularly in the field of oncology.

NGS a rapidly developing technology experiencing decreases in costs that make it an affordable choice for genetic testing in cancer [11-13]. Several NGS assays have been approved as in vitro diagnostics, with more being developed. These include targeted and whole exome sequencing (WES); for example, tests from FMI, NanthHealth and Thermo Fisher [14-16].

Patient samples in cancer clinical trials are routinely used for WES genomic profiling. Fresh-frozen tissue is the optimal and preferred source of DNA for WES assay, but it is not always available. More readily available tissue samples, such as formalin-fixed paraffinembedded (FFPE) tissues, are more challenging to work with. A range of optimized protocols is used to overcome the challenge of processing FFPE samples to obtain good quality DNA and quality sequencing data [17]. WES analytical sequencing metrics are regularly used to evaluate the quality of NGS data. Routine WES quality evaluation can be informative about the performance of clinical laboratories 


\begin{tabular}{|c|c|c|c|c|c|}
\hline Samples & Total & Pass & Fail & Pass (\%) & Fail (\%) \\
\hline \multicolumn{6}{|l|}{ WES samples } \\
\hline - WES samples & 12,527 & 11,108 & 1419 & 88.7 & 11.3 \\
\hline - Tumor & 6561 & 6062 & 498 & 92.4 & 7.6 \\
\hline - Normal & 5966 & 5960 & 6 & 99.9 & 0.1 \\
\hline \multicolumn{6}{|l|}{ WES sets } \\
\hline - WES sets & 6561 & 5836 & 725 & 88.9 & 11.1 \\
\hline - TN sets & 5966 & 5272 & 694 & 88.4 & 11.6 \\
\hline - TO sets & 595 & 564 & 31 & 94.8 & 5.2 \\
\hline \multicolumn{6}{|c|}{ Cohort matcher QC } \\
\hline - TN sets & 5966 & 5707 & 259 & 95.7 & 4.3 \\
\hline
\end{tabular}

QC: Quality control; TN: Tumor-normal pair; TO: Tumor only; WES: Whole exome sequencing.

and can help to improve tissue collection and processing protocols. Here we utilized the WES QC metrics and genetic matching to assess the quality of data generated from samples collected across multiple clinical trial sites.

\section{Materials \& methods}

All clinical data were collected as part of each clinical trial according to the institutional board ethics guidelines. This study used quality metrics from the clinical trial WES results, but not the results themselves.

\section{WES sample preparation}

The WES libraries were performed according to the standard protocols described by Chang et al. [18]. Downstream data processing (from the raw FASTQ data to BAM files) was performed and the QC metrics were generated based on the Broad Institute's best practice recommendations [19] and according to the Sentieon WES processing pipeline described by Chang et al. [18].

\section{Cohort matcher}

The tumor and the normal samples were processed to generate individual tumor and normal BAM files. The Bristol Myers Squibb (BMS) cohort matcher tool [20] was developed from the public tool BAM-matcher [21]. The cohort matcher compared BAMs from the tumor and blood and measured the percentage match of the germline variants of both WES samples. A set of $\sim 7500$ loci of common variants present in the germline with population frequency between 0.3 and 0.5 was used for the cohort matcher genotype matching. Those tumor-normal (TN) pairs with genotype match less than 0.85 were marked as 'QC cohort failed' and used to identify sample swaps and large contamination issues. The cohort QC results were used for the clinical laboratories' performance evaluation.

\section{Model for the site performance analysis}

Modeling of relative site performance was done based on 166 sites that processed $>10$ samples. The model was developed in $\mathrm{R}$ using package 'arm' v1.10-1. Each site's odds ratio (OR) was evaluated relative to a reference site - a site with the median QC success rate. The code for the model and statistical analysis is available in GitHub [22].

\section{Results \& discussion}

\section{WES QC metrics}

Clinical research laboratories are the heart of the translational research for development of cancer drugs and personalized medical care. Laboratories collect and process solid tumor biopsies which are used for the NGS WES assay and other tests. For a large organization such as BMS, it is important to keep track of the performance of participating laboratories across the globe. In this retrospective study, we explored WES metrics as quality indicators for performance assessment of the sample collecting sites.

A WES assay was used for patients in cancer clinical trials to evaluate tumor-specific somatic variants and DNA structural changes [23]. For an accurate analysis, WES results were generated from the solid tumor tissue and from the corresponding normal or blood sample. We performed QC analysis of 12,527 WES results: 6561 WES results obtained from the solid tumor tissue and 5966 WES results from blood samples (Table 1). These samples were collected by 598 medical laboratories worldwide, for 29 different trials and 37 indications. 95\% of the tumor tissue samples were FFPE samples. All samples were evaluated by the presequencing QC step, which removed samples with low DNA yields (5.5\%) and therefore enriched for the quality samples for WES assay. The samples were handled uniformly across all sites and were shipped on dry ice to mitigate the impact of storage and shipping on sample quality. The presequencing and sequencing processes were performed at the same vendor for all samples based on the same standard protocol and the same capture kit as described by Chang et al. [18]. Therefore the quality assessment of WES results represents mostly site-specific differences in sample collection. 


\begin{tabular}{|c|c|c|c|c|}
\hline Indication & Total (n) & Total (\%) & Failed (n) & Failed (\%) \\
\hline Non-small-cell lung cancer & 1614 & 25.5 & 112 & 6.9 \\
\hline Melanoma & 1552 & 24.5 & 134 & 8.6 \\
\hline Renal cell carcinoma & 1282 & 20.2 & 109 & 8.5 \\
\hline Small-cell lung cancer & 430 & 6.8 & 20 & 4.7 \\
\hline Colorectal cancer & 271 & 4.3 & 10 & 3.7 \\
\hline Hepatocellular carcinoma & 219 & 3.5 & 29 & 13.2 \\
\hline Bladder cancer & 189 & 3 & 28 & 14.8 \\
\hline Glioblastoma & 145 & 2.3 & 2 & 1.4 \\
\hline Head and neck cancer & 131 & 2.1 & 2 & 1.5 \\
\hline Gastroesophageal junction cancer & 86 & 1.4 & 12 & 14 \\
\hline Pancreatic cancer & 77 & 1.2 & 0 & 0 \\
\hline Heme & 74 & 1.2 & 1 & 1.4 \\
\hline Ovarian cancer & 66 & 1 & 0 & 0 \\
\hline Prostate cancer & 65 & 1 & 8 & 12.3 \\
\hline Cervical carcinoma & 21 & 0.3 & 0 & 0 \\
\hline Other indications $(\mathrm{n}<20)$ & 113 & 1.8 & 3 & 2.7 \\
\hline
\end{tabular}

The sequencing quality of WES results was evaluated using the Picard sequencing QC toolkit $[19,23]$. The sequencing QC metrics evaluated for each sample included total reads $>45$ million, mean coverage of the targeted region $>50 \times$ and percentage of targeted region with at least $20 \times$ coverage $>80 \%$. A sample sequencing QC was considered failed if at least one of these criteria was not met. We processed a total of 6561 tumor and $5966 \mathrm{normal}$ samples for WES assay. The normal samples were from blood; only a small percentage of them $(0.1 \%)$ failed sequencing QC. The tumor samples were FFPE solid tumor samples and, as expected, experienced a higher failure rate, at $7.6 \%$ (Table 1).

We hypothesized that indication type may have an effect on the quality of the extracted DNA and influence the WES QC results for the tumor samples. We therefore evaluated WES QC percentage failure for each indication. The BMS clinical trials focused on several indications; non-small-cell lung cancer, melanoma and renal cell carcinoma comprised $71.2 \%$ of all WES results (Table 2). Head and neck cancer, pancreatic cancer, heme, ovarian cancer and cervical carcinoma had relatively low percentage failure rates $(0-5 \%)$. Prostate cancer, hepatocellular carcinoma, bladder cancer and gastroesophageal junction cancer resulted in higher failure rates $(>12 \%)$, suggesting that these indications were more challenging for the WES assay (Table 2). Thus it is necessary to account for the indication when performing a quality assessment of a clinical site using WES QC criteria.

\section{Cohort matching as a quality indicator for the clinical laboratory's performance}

The NGS best practices for tumor variant calling using WES data recommend using TN paired sequencing WES sets for the best downstream analysis [19,24]. To ensure that both tumor and blood (normal) samples were derived from the same individual, we developed a cohort matcher QC $[20,21]$. The cohort matcher QC method measured the percentage of matched germline variants between the tumor and the blood WES results in order to verify that both samples were collected from the same patient. Based on the recommendations of Wang et al. [21] and inspection of the full dataset, we used a cutoff of $>85 \%$ genetic match for a TN pair to pass cohort QC.

A combination of tumor sequencing QC, normal sequencing QC and the cohort QC were used to assess the quality of a TN set - a unit of results per patient for the WES data analysis. The TN paired samples comprised the majority (91\%) of all WES results; $88.9 \%$ of these TN paired sets passed all WES QC steps (Table 1). 498 tumor samples (7.6\%) showed suboptimal sequencing outcomes, and 259 TN sets (4.3\%) failed cohort matcher pairing QC (Table 1). Only 39 of the TN failed cohort QC sets were samples that also failed tumor sequencing QC (Figure 1A).

Based on the overall distribution of the percentage of genetic match and results from Wang et al. [21], we characterized the TN sets into three categories of cohort-matching QC outcomes: matched, nonmatched and contaminated (Figure 1B). TN sets with greater than $85 \%$ genetic match were defined as matched TN and were called 'passed cohort QC'; the distribution of percentage genetic matching for these samples showed a strong peak at $98 \%$. The contaminated or low-confidence samples were TN sets that enriched at the $81 \%$ peak of genetic matching. These were patients with less than $85 \%$ genetic match and the best predicted TN pair both came from the same patient (Figure 1B). TN sets enriched at the $43 \%$ peak (Figure 1B) had less than $85 \%$ genetic matching, but exhibited a better percentage match with a sample from another patient; therefore these were defined as nonmatched or potential swap events.

To uncover true sample swaps, the nonmatched TN sets with only tumor and normal samples that passed sequencing QC were used. We identified 51 cross-patient alternative TN sets with greater than $85 \%$ genetic matching and labeled these as high-confidence sample swap events. However, $63 \mathrm{TN}$ samples failed to find a high-confidence matching alternative pair, perhaps because not all samples were 
(A)

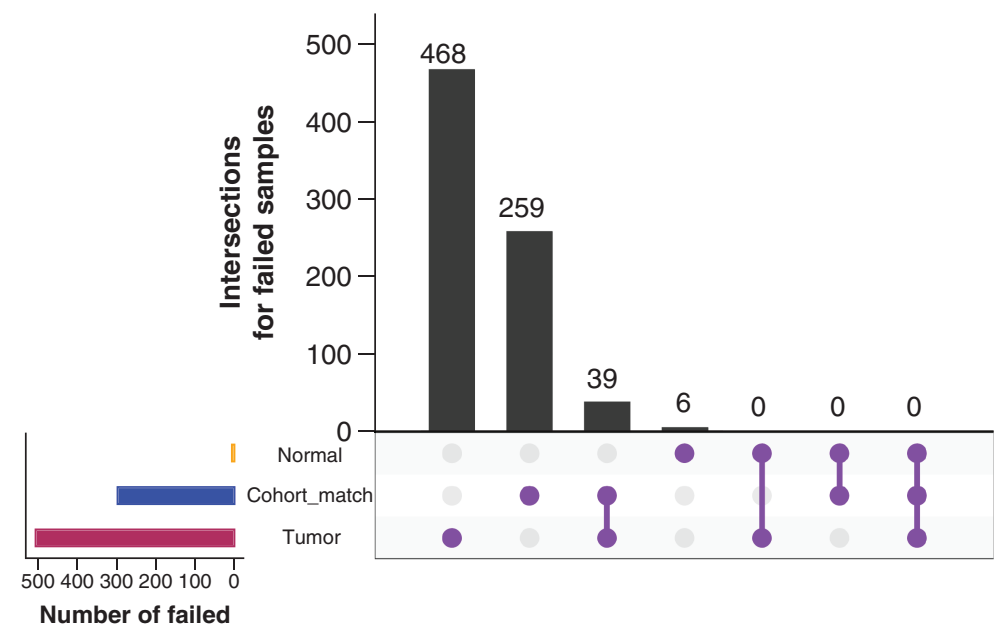

(B)

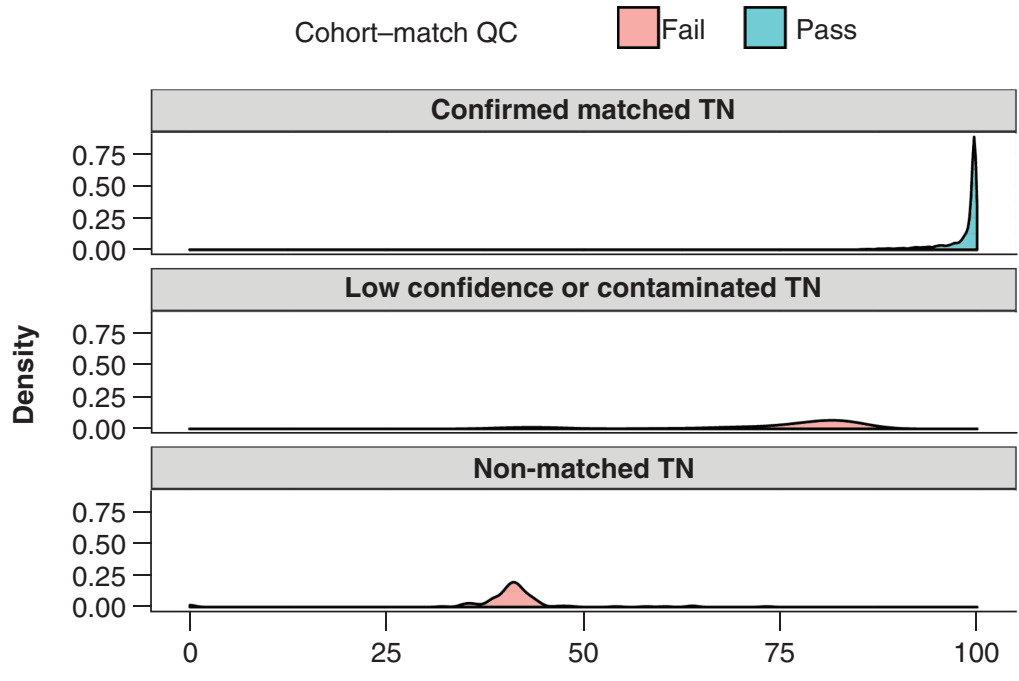

Percent of genetic match
(C) Swap identification

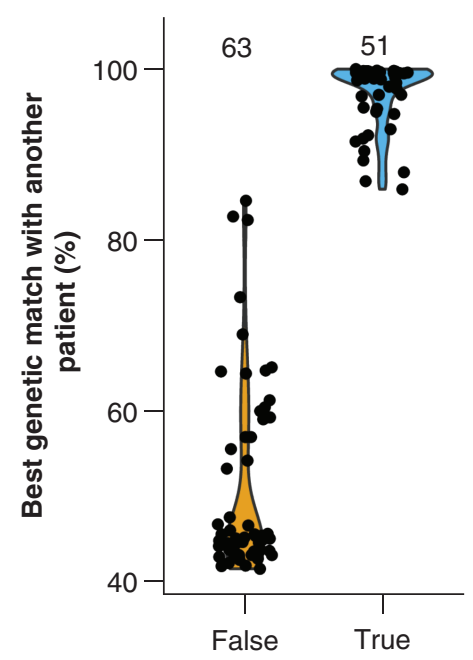

High confidence swap

Figure 1. Characterization of the WES quality control results for the tumor-normal pairs. (A) The intersection of QC outcomes for failed TN sets by the sequencing results of normal (blood) samples, the sequencing results of tumor samples and the TN genetic matching of cohort-match QC. We observed an intersection of 39 samples that failed both tumor sequencing QC and cohort-match QC, but none for other intersections. (B) Cohort-match QC allows the identification of three categories of TN samples: matched (passed QC), contaminated (failed QC) and nonmatched (failed QC). (C) Identification of sample swap in the 'QC failed' nonmatched TN sets. A cut-off value of $\geq 85 \%$ of genetic match with a sample from another patient was used to identify the high-confidence swap events.

QC: Quality control; TN: Tumor-normal pair.

used for WES assay (Figure 1C). Although the cohort-matching QC step can verify genetic pairing, there is often not enough information to find the source of contamination or to correct sample swaps.

\section{Modeling site performance}

A combination of tumor sequencing QC, normal sequencing QC and the cohort-matching QC was used to develop a modeling approach for the assessment of relative site performance. We used Bayesian logistic regression to model the relationship between QC success rate and site. We compared two models: one adjusting for indication and one without adjusting for indication. Because many clinical labs processed only small numbers of samples for BMS clinical studies, we performed the analysis using data from 166 sites which had processed more than ten samples. 
(A)

Estimates of success for selected sites

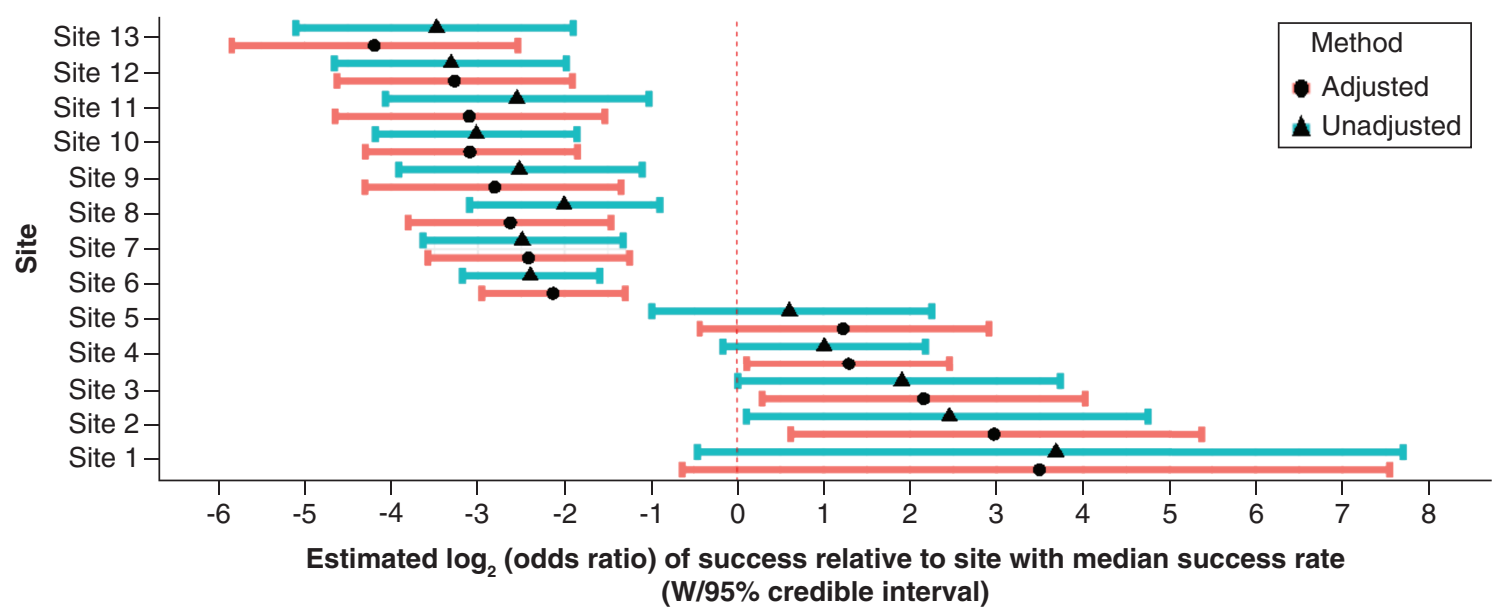

(B)

Distribution of estimates of success across all sites

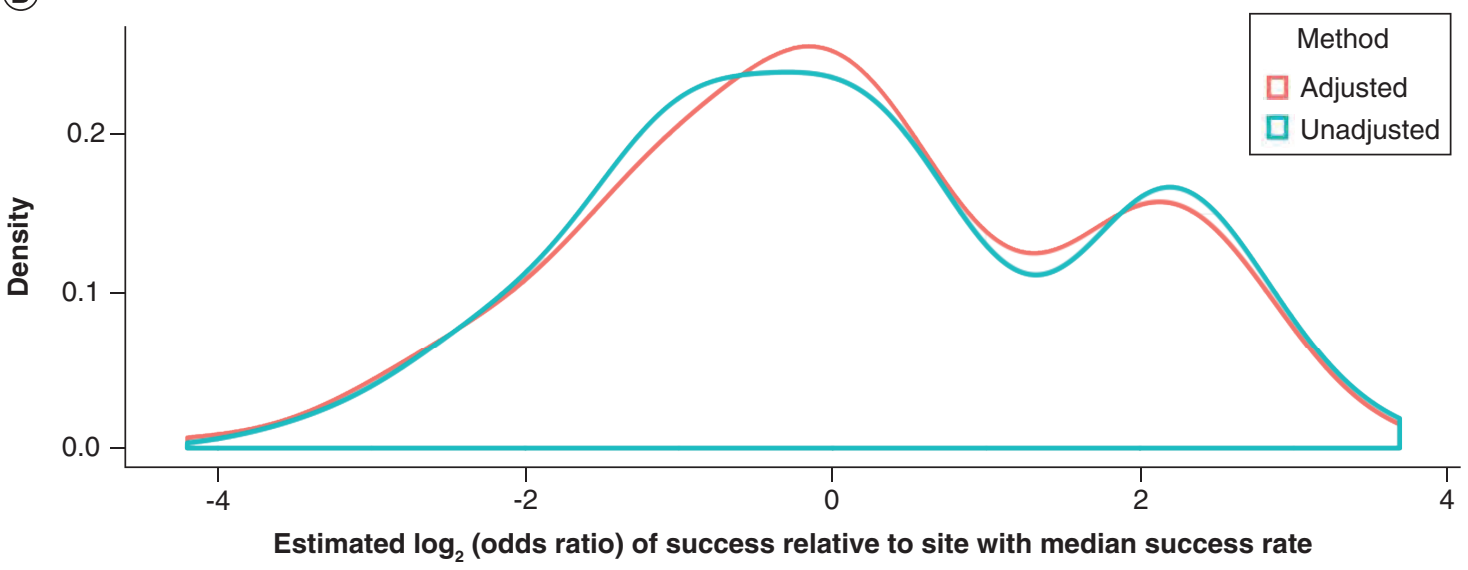

Figure 2. Modeling results of the relative site performance. (A) Estimated success for the selected sites. The estimated log2(Odds Ratio) for each site with $95 \%$ credible intervals for the model adjusted by the indication is shown in red and unadjusted is shown in blue. (B) Distribution of the estimated success across all sites colored by the model; red is adjusted by indication and blue is the unadjusted model.

In order to produce results that reflected performance of a particular site irrespective of the indication, we assessed performance based on each site's OR relative to a reference site. We chose the site with the median success rate as the reference site in order to make interpretation of the ORs more intuitive.

We used Bayesian logistic regression (as opposed to frequentist logistic regression) in order to avoid a separation problem in which some sites had either all successes or all failures, which would make it impossible to have stable results. By applying Bayesian logistic regression, we were able to stabilize the results [25].

After running the model, we were able to produce each site's OR of success relative to the chosen reference site with $95 \%$ credible intervals. We were then able to differentiate site performance based on median OR adjusted by the indication. We categorized sites with $\log 2(\mathrm{OR})<-2$ and the credible interval of $<-1$ as 'poor performers' and sites with $\log 2(\mathrm{OR})>1$ as 'good performers' (Figure 2A).

Out of the 166 sites in our model, we identified 8 which were clearly poor performers and 5 sites which were clearly good performers (Figure 2A). Overall, the log2(OR) ranged from -4.2 to 3.7. The distribution of the log2(OR) (Figure 2B) for adjusted (for indication) and nonadjusted models showed two peaks. The right distribution corresponded to the 'very good performers' cluster and the left distribution corresponded to the 'average performers'. Sites that had log2(OR) closer to -4.2 corresponded to the 'very poor performers'.

The overall distribution showed little difference in the estimated OR from the adjusted and nonadjusted models (Figure 2B). Adjusting for the indication rewarded some sites that worked with more challenging tissues and vice versa. Such movement is not visible in the overall density plot for the adjusted versus unadjusted log2(OR) of the site's success rate (Figure 2B). However, at the level of individual 
sites there were several sites with notable differences (Figure 2A). In particular, three out of the eight sites labeled poor performers had a difference in the $\log 2(\mathrm{OR})>0.5$. Similarly, two out of the five sites labeled good performers had a difference in the log2(OR) $>0.5$.

Modeling site performance based on WES QC metrics for tumor and blood samples, and cohort-matching QC can be used to evaluate clinical site performance and provide a guidance for business decisions.

We developed and described a flexible modeling approach that can account for the most important confounding factors. Here we evaluated the impact of indication on performance. Other factors that might be worth investigating could include sample age, sample format (FFPE block or slide) and other preanalytical variables. It is possible that the failure differences of indications were influenced by additional hidden factors not accounted for in this analysis. Thus the method we describe is a first step in the process of evaluating clinical sites' performance and adherence to best practices. Follow-up of these initial findings through review of the sites' practices and on-site visits would be critical for validation of the results and addressing potential deficiencies.

The cohort-matching QC step can help to identify clinical sites that may need to improve their sample-tracking procedures to prevent sample swaps. Additional measures to reduce cross-contamination need to be taken at the collection site, along the path of the sample shipment and during postprocessing.

\section{Future perspective}

This study demonstrates how the WES QC process can be used as a method to validate sample quality and site performance. As WES data from additional subjects become available, we expect to be able to increase the power of this analysis, evaluate additional sites and track performance longitudinally. Expanding the sample collection information (e.g., shipment of slides vs blocks) will further help us identify potential sources of failure. We expect that insights gained from these performance metrics will catalyze site-specific discussions and optimization plans that will yield improved sample and data quality, thereby magnifying the impact that NGS-based assays can have on the discovery and application of biomarkers.

\section{Author contributions}

N Kalinava, R Cartmell, S Srinivasan, R Golhar and A Sasson designed the computational framework. N Kalinava performed data analysis. A Apfel developed the model. M Chien and K Kim verified the calculations. K Bednarz and S Pant contributed to data collection. N Kalinava and S Kirov wrote the manuscript with input from all authors. J Szustakowski, S Chasalow, A Sasson and S Kirov were in charge of overall direction.

\section{Acknowledgments}

We thank A Greenfield, D Huston, D Reinhard and P Narasimhan for providing information about the clinical sites and the involved patients for providing the sequencing data used in this study.

\section{Financial \& competing interests disclosure}

The research for this paper was funded by Bristol Myers Squibb. All of the authors have no conflicts of interest to declare beyond being BMS employees and receiving compensation from the company. The authors have no other relevant affiliations or financial involvement with any organization or entity with a financial interest in or financial conflict with the subject matter or materials discussed in the manuscript apart from those disclosed.

No writing assistance was utilized in the production of this manuscript.

\section{Ethical disclosure}

The authors state that they have obtained appropriate institutional review board approval or have followed the principles outlined in the Declaration of Helsinki for all human or animal experimental investigations. In addition, for investigations involving human subjects, informed consent has been obtained from the participants involved.

\section{Open access}

This work is licensed under the Attribution-NonCommercial-NoDerivatives 4.0 Unported License. To view a copy of this license, visit http: //creativecommons.org/licenses/by-nc-nd/4.0/

\section{References}

1. NCI Best Practices for Biospecimen Resources. 2011. https://biospecimens.cancer.gov/bestpractices/2011-NCIBestPractices.pdf

2. World Health Organization, Special Programme for Research and Training in Tropical Diseases. Good clinical laboratory practice (GCLP). [Internet]. World Health Organization, Geneva, Switzerland, http://apps.who.int/tdr/svc/publications/tdr-research-publications/gclp-web

3. European Medicines Agency. European Medicines Agency. https://www.ema.europa.eu/en

4. Clinical Research Regulation For China | ClinRegs. https://clinregs.niaid.nih.gov/country/china\#_top

5. Clinical Research Regulation For India | ClinRegs. https://clinregs.niaid.nih.gov/country/india\#_top

6. Rubin HR, Pronovost P, Diette GB. From a process of care to a measure: the development and testing of a quality indicator. Int. J. Qual Health Care 13(6), 489-496 (2001).

7. Aziz N, Zhao Q, Bry L et al. College of American Pathologists' Laboratory Standards for Next-Generation Sequencing Clinical Tests. Arch. Pathol. Lab. Med. 139(4), 481-493 (2014). 
8. Matthijs G, Souche E, Alders M et al. Guidelines for diagnostic next-generation sequencing. Eur. J. Hum. Genet. 24(1), 2-5 (2016).

9. GA4GH [Internet]. https://www.ga4gh.org/

10. WHO Tools and resources. WHO. http://www.who.int/genomics/elsi/en/

11. Beigh MM. Next-generation sequencing: the translational medicine approach from 'bench to bedside to population.. Medicines (Basel) $3(2), 14$ (2016)

12. Muir P, Li S, Lou S et al. The real cost of sequencing: scaling computation to keep pace with data generation. Genome Biol. 17(1), 53 (2016).

13. Bewicke-Copley F, Arjun Kumar E, Palladino G, Korfi K, Wang J. Applications and analysis of targeted genomic sequencing in cancer studies. Comput. Struct. Biotechnol. J. 17, 1348-1359 (2019).

14. Commissioner of the FDA announces approval, CMS proposes coverage of first breakthrough-designated test to detect extensive number of cancer biomarkers. FDA (2020). http://www. fda.gov/news-events/press-announcements/fda-announces-approval-cms-proposes-coverage-first-breakthrough-designated-test-detect-extensive

15. NantHealth's TMB Assay Wins FDA Authorization. Clinical OMICs - Molecular Diagnostics in Precision Medicine (2019). https://www.clinicalomics.com/news-and-features/nanthealthsomics-core-tmb-assay-wins-fda-authorization/

16. Oncology Diagnostics - US. https://www.thermofisher.com/us/en/home/clinical/diagnostic-testing/condition-disease-diagnostics/oncology-diagnostics.html

17. Astolfi $A$, Urbini $M$, Indio $V$ et al. Whole exome sequencing (WES) on formalin-fixed, paraffin-embedded (FFPE) tumor tissue in gastrointestinal stromal tumors (GIST). BMC Genomics 16, 892 (2015).

18. Chang H, Sasson A, Srinivasan S et al. Bioinformatic methods and bridging of assay results for reliable tumor mutational burden assessment in non-small-cell lung cancer. Mol. Diagn. Ther. 23(4), 507-520 (2019).

19. McKenna A, Hanna M, Banks E et al. The Genome Analysis Toolkit: a MapReduce framework for analyzing next-generation DNA sequencing data. Genome Res. 20(9), 1297-1303 (2010).

20. Golhar R. golharam/cohort-matcher. https://github.com/golharam/cohort-matcher

21. Wang PPS, Parker WT, Branford S, Schreiber AW. BAM-matcher: a tool for rapid NGS sample matching. Bioinformatics 32(17), 2699-2701 (2016).

22. Github. https://github.com/apfela2/Site_QC

23. Shi W, $\mathrm{Ng} \mathrm{CKY,} \mathrm{Lim} \mathrm{RS} \mathrm{et} \mathrm{al.} \mathrm{Reliability} \mathrm{of} \mathrm{whole-exome} \mathrm{sequencing} \mathrm{for} \mathrm{assessing} \mathrm{intratumor} \mathrm{genetic} \mathrm{heterogeneity.} \mathrm{Cell} \mathrm{Reports} \mathrm{25(6),} \mathrm{1446-1457} \mathrm{(2018).}$

24. Picard Tools By Broad Institute. https://broadinstitute.github.io/picard/index.html

25. Gelman A, Carlin JB, Stern HS et al. Bayesian Data Analysis. Chapman and Hall/CRC. https://www.taylorfrancis.com/books/9780429113079 
